\title{
VARIÂNCIAS DO PONTO CRÍTICO DE EQUAÇÕES DE REGRESSÃO QUADRÁTICA
}

\author{
Variances of the critical point of a quadratic regression equation
}

\author{
Ceile Cristina Ferreira Nunes ${ }^{1}$, Augusto Ramalho de Morais ${ }^{2}$, Joel Augusto Muniz ${ }^{3}$, Thelma Sáfadi ${ }^{2}$
}

\section{RESUMO}

Com o presente trabalho teve-se por objetivo a determinação de variâncias para o estudo do ponto crítico de uma equação de regressão de segundo grau, em situações experimentais com diferentes variâncias, por meio de simulação Monte Carlo. Em muitos estudos, teóricos ou aplicados, o pesquisador depara-se com o problema que envolve quociente entre variáveis aleatórias e, principalmente, entre variáveis normais. Como exemplo, aquelas que surgem em pesquisas de dose econômica de nutrientes em experimentos de adubação, de compactação de solos e em outros problemas em que há interesse na variável aleatória $\hat{x}=\hat{b} /(-2 \hat{c})$, estimador do ponto crítico na regressão $\hat{y}=\hat{a}+\hat{b} x+\hat{c} x^{2}$. Para estudar a distribuição do ponto crítico de uma equação de regressão quadrática, foram utilizados dados de produção de algodão de 536 ensaios, ajustando-se um modelo quadrático. A estimação dos parâmetros foi feita pelo método dos quadrados mínimos ordinários. Com base nessas estimativas, implementou-se por meio do software MATLAB ${ }^{\circledR}$ uma rotina para simulação de duas séries com 5000 erros aleatórios de distribuição normal de média zero relativos a cada uma das variâncias consideradas teóricas: $\sigma^{2}=0,1 ; 0,5 ; 1 ; 5 ; 10$; 15; 20 e 50. As estimativas da variância do ponto crítico foram obtidas por meio de três métodos: (a) fórmula comum do cálculo de variâncias; (b) fórmula obtida pela diferenciação do estimador do ponto crítico e (c) fórmula demonstrada para o cálculo da variância de uma razão, considerando-se a covariância entre $\hat{b}$ e $\hat{c}$. Pelos resultados obtidos para as estatísticas médias dos coeficientes de regressão $\hat{b}$ e $\hat{c}$, bem como suas respectivas variâncias em função das diversas variâncias teóricas $\left(\sigma^{2}\right)$ adotadas, verificou-se que esses valores teóricos estão próximos aos reais. Ainda ocorre uma tendência de que, com o aumento da variância teórica, esses valores aumentem. Pode-se concluir que a variância do ponto crítico calculada usando-se a expressão que leva em consideração a covariância entre $\hat{b}$ e $\hat{c}$ apresenta resultados mais satisfatórios e que não segue uma distribuição normal, pois apresenta uma distribuição de freqüência com assimetria positiva e formato leptocúrtico.

Termos para indexação: Regressão quadrática, quociente de variáveis aleatórias, variância do ponto crítico, intervalo de confiança.

\section{ABSTRACT}

The aim of this paper is determine variances for the analysis of the critical point of a second-degree regression equation in experimental situations with different variances through Monte Carlo simulation. In many theoretical or applied studies, one finds situations involving ratios of random variables and more frequently normal variables. Examples are provided by variables, which appear in economic dose research of nutrients in fertilization experiments, as well as in other problems in which there are interests in the random variable $\hat{x}=\hat{b} /(-2 \hat{c})$, estimator of the critic point in the regression $\hat{y}=\hat{a}+\hat{b} x+\hat{c} x^{2}$. Data of five hundred thirty six trials in cotton yield were utilized to study the distribution of the critical point of a quadratic regression equation by adjusting a quadratic model. The parameters were evaluated using a least square method. From the estimations a MATLAB routine was implemented to simulate two sets with five thousands random errors with normal distribution and zero mean, relative to each of the theoretical variances: $\sigma^{2}=0.1 ; 0.5 ; 1 ; 5 ; 10 ; 15 ; 20$ and 50 . The estimation of the variance of the critical point was obtained by three methods: (a) usual formula for the variance; (b) formula obtained by differentiation of the critical point estimator and (c) formula for the computation of the variance of a quotient by taking into consideration the covariance between $\hat{b}$ and $\hat{c}$. The results obtained for the statistic average for the regression

(Recebido para publicação em 23 de abril de 2002 e aprovado em 8 de agosto de 2002)

\footnotetext{
1. Mestre em Estatística e Experimentação Agropecuária - Universidade Federal de Lavras/UFLA - Caixa Postal 37 - $37200-000$ Lavras, MG

2. Professores Adjunto do Departamento de Ciências Exatas/UFLA.

3. Professor Titular do Departamento de Ciências Exatas/UFLA.
} 
between $\hat{b}$ e $\hat{c}$, as well as its respective variances in terms of the several theoretical residual variances $\left(\sigma^{2}\right)$ adopted show that those theoretical values are close to real ones. Moreover, there is a trend of increasing $\hat{b}$ and $\hat{c}$ with increase of the theoretical variance. It may be concluded that the critical point variance calculated taking into consideration the covariance between $\hat{b}$ and $\hat{c}$, gives more satisfactory results and does not follow a normal distribution, presenting a frequency distribution with positive assimetry and leptokurtic shape.

Index terms: Quadratic regression, quotient random variables, variance of the critical point, interval of confidence.

\section{INTRODUÇÃO}

Entre os vários modelos de regressão usados nas pesquisas, merece destaque o modelo de regressão quadrático, devido ao seu amplo uso, facilidade de cálculos e enorme adaptação na explicação e interpretação de fenômenos biológicos, como nas pesquisas agropecuárias, com o uso de fertilizantes. Seja o modelo de regressão quadrática, com uma variável independente, dado por Neter et al. (1990) por $\hat{y}_{i}=\hat{a}+\hat{b} x_{i}+\hat{c} x_{i}^{2}$, pode-se obter um estimador para o ponto crítico dessa equação, conforme Kaplan (1972), derivando-se $\hat{y}_{i}$ em relação a $x$ e fazendo sua derivada igual a zero, assim:

$$
\frac{d \hat{y}_{i}}{d x}=\frac{d\left(\hat{a}+\hat{b} x_{i}+\hat{c} x_{i}^{2}\right)}{d x}=\hat{b}+2 \hat{c} x_{i}=0
$$

logo, o estimador do ponto crítico da equação de regressão quadrática é $\hat{x}_{i}=\frac{\hat{b}}{-2 \hat{c}}$, que será a abscissa de um ponto de máximo, se $\hat{C}$ for negativo, e de mínimo, se $\hat{C}$ for positivo.

\section{Estimativa da variância do ponto crítico}

O conhecimento da variância de um ponto crítico pode ser de muita importância em algumas pesquisas, pois possibilita construir intervalos de confiança para o verdadeiro valor do ponto crítico e testar sua validade ou não por meio de uma hipótese de interesse a seu respeito.

Para estimar a variância do ponto crítico em um estudo usando simulação, D’Aulísio (1976) utilizou a expressão usual

$$
\operatorname{Var}(\hat{x})=\frac{\sum_{i=1}^{n} x_{i}^{2}-\frac{\left(\sum_{i=1}^{n} x_{i}\right)^{2}}{n}}{n-1},
$$

citada por Spiegel (1993). Segundo Mood et al. (1974), a variância de um quociente de duas variáveis aleatórias $X$ e $y$ é dada de forma aproximada pela expressão:

$$
\operatorname{Var}\left[\frac{x}{y}\right] \approx\left(\frac{\mu_{X}}{\mu_{Y}}\right)^{2}\left(\frac{\operatorname{Var}[x]}{\mu_{X}^{2}}+\frac{\operatorname{Var}[y]}{\mu_{Y}^{2}}-\frac{2 \operatorname{Cov}[x, y]}{\mu_{X} \mu_{Y}}\right) .
$$

Aulísio (1976), estudando a variância dos pontos de máximo ou de mínimo de equações de regressão de segundo grau, demonstrou que se $x=\frac{-\hat{b}}{2 \hat{c}}$ é um estimador consistente do ponto crítico, sua variância pode ser estimada aproximadamente pela primeira derivada do estimador do ponto crítico em relação a $X$, e, portanto, o estimador da variância é dado pela expressão:

$$
\operatorname{Var}(\hat{x})=\frac{1}{4}\left[\frac{\operatorname{Var}(\hat{b})}{\hat{c}^{2}}+\frac{\hat{b}^{2} \cdot \operatorname{Var}(\hat{c})}{\hat{c}^{4}}\right]
$$

\section{Coeficientes de assimetria e curtose}

Testes que avaliam assimetria e curtose são úteis e ajudam a verificar a significância da variação de certos momentos da amostra, em relação aos valores esperados, de uma população normal. Nas aplicações práticas, porém, podem-se ter valores próximos de zero e, principalmente, neste caso, existe a necessidade de saber se o valor obtido é diferente ou não de zero.

O coeficiente de assimetria pode ser estimado, conforme definido por Fisher (1930), usando-se a expressão $\hat{\gamma}_{1}=\frac{\hat{\mu}_{3}}{\hat{\sigma}^{3}}$. Para verificar se os valores estimados de $\hat{\gamma}_{1}$ diferem ou não de zero, esse pesquisador sugeriu a aplicação do teste de "t" de Student $t=\frac{\hat{\gamma}_{1}-\gamma_{1}}{s\left(\hat{\gamma}_{1}\right)}$, em que o valor da estatística " $t$ " é a razão entre a estimativa do parâmetro $\left(\gamma_{1}\right)$ e seu erro-padrão, obtido a 
partir da raiz quadrada da variância $\operatorname{Var}\left(\hat{\gamma}_{1}\right)$, dada por $\operatorname{Var}\left(\hat{\gamma}_{1}\right)=\frac{6 n(n-1)}{(n-2)(n+1)(n+3)}$.

$\mathrm{O}$ coeficiente de curtose foi definido por de Fisher (1930) como $\hat{\gamma}_{2}=\frac{\hat{\mu}_{4}}{\hat{\sigma}^{4}}-3$. Para verificar se os valores estimados de $\hat{\gamma}_{2}$ diferem ou não de zero, aplica-se o teste "t" dado por $t=\frac{\hat{\gamma}_{2}-\gamma_{2}}{s\left(\hat{\gamma}_{2}\right)}$ para o qual $\operatorname{Var}\left(\hat{\gamma}_{2}\right)=\frac{24 n(n-1)^{2}}{(n-3)(n-2)(n+3)(n+5)}$.

\section{Intervalos de confiança}

Um método para se obter intervalo de confiança para um quociente é o baseado no teorema de E.C. Fieller, Finney (1964).

Sendo $x_{1}$ e $x_{2}$ duas variáveis aleatórias normalmente distribuídas com média zero e variância $\sigma_{1}^{2}$ e $\sigma_{2}^{2}$, respectivamente, a nova variável $u=x_{1}-\mu x_{2}$, função linear das observações $x_{1}$ e $x_{2}$, terá também distribuição normal de média zero e variância $\operatorname{Var}(u)=\sigma_{1}^{2}+\mu^{2} \sigma_{2}^{2}$.

Sendo $s_{1}^{2}$ e $s_{2}^{2}$ estimadores não-viesados de $\sigma_{1}^{2}$ e de $\sigma_{2}^{2}$, respectivamente, o quociente:

$$
\frac{u}{\sqrt{\operatorname{Var}(\hat{u})}}=\frac{x_{1}-\mu x_{2}}{\sqrt{s_{1}^{2}+\mu^{2} s_{2}^{2}}},
$$

tem distribuição "t" de Student (KENDALL e STUART, 1977).

Assim sendo, em um certo nível de probabilidade $\alpha$ apropriado, a inequação $\left(x_{2}^{2}+t^{2} s_{2}^{2}\right) \mu^{2}-2 x_{1} x_{2} \mu+x_{1}^{2}-t^{2} s_{1}^{2} \leq 0$ dá os extremos do intervalo de confiança.

\section{MATERIAL E MÉTODOS}

\section{Material}

Os dados utilizados neste trabalho foram os mesmos utilizados por Freitas (1978), e são provenientes de 536 ensaios conduzidos com a cultura de algodão, realizados nos Estados do Pa- raná, Minas Gerais e Goiás, no período de 1970/1975.

\section{Métodos}

Este trabalho foi realizado por meio de simulação Monte Carlo, para avaliar distribuições de freqüências do ponto crítico de uma equação de regressão do segundo grau. Consideraram-se como referência os valores médios obtidos por Freitas (1978) para os coeficientes de regressão cujas estimativas foram $\hat{b}=90,1713$ e $\hat{c}=-98,8947$, necessárias para o cálculo do ponto crítico, e considerando que as variâncias eram proporcionais, sendo $\operatorname{Var}(\hat{c})=24,4532 \operatorname{Var}(\hat{b}) . \quad$ Para simular dados experimentais para $\hat{b}$ e $\hat{c}$, com média e variância estipulados conforme a situação, foi desenvolvido um procedimento utilizando o software MATLAB ${ }^{\circledR}$ - Matrix Laboratory. Consideraram-se as seguintes dosagens com 4 repetições cada uma: $0.0 ; 0.2 ; 0.4 ; 0.6$ e 0.8 , caracterizadas pelas diferentes variâncias teóricas, $\sigma^{2}=0,1 ; 0,5 ; 1,0 ; 5,0$; $10 ; 15 ; 20 ; 50$. A partir desses valores reais, geraram-se duas séries com 5000 erros aleatórios cada uma, com distribuição normal de média zero para cada uma das variâncias teóricas consideradas, denominando $e_{1}$ a primeira série e $e_{2}$ a segunda, de forma que $e_{1} \sim N\left(0, \sigma^{2}\right)$ e $e_{2} \sim N\left(0, \sigma^{2}\right)$.

Obtiveram-se, então, 5000 erros $e_{3}$ após multiplicar e $\mathrm{e}_{2}$ por $\sqrt{24,4532}$; assim, as estimativas de $\hat{b}$ e $\hat{c}$ podem ser escritas como $\hat{b}=90,1713+e_{1} \quad e \quad \hat{c}=-98,8947+e_{2}$. Assim, construiu-se um vetor com 5000 observações, cujos elementos são $\hat{x}=\frac{\hat{b}}{-2 \hat{c}}$. Determinaram-se então, os valores médios para as estimativas $\hat{b}, \hat{c}$ e $\hat{x}$, suas respectivas variâncias com a finalidade de verificar a qualidade da simulação.

A variância do quociente ou do ponto crítico $\hat{x}=\frac{\hat{b}}{-2 \hat{c}}$ foi calculada utilizando-se três expressões $\operatorname{Var}_{1}(\hat{x}), \operatorname{Var}_{2}(\hat{x})$ e $\operatorname{Var}_{3}(\hat{x})$, a saber: 


$$
\begin{gathered}
\operatorname{Var}_{1}(\hat{x})=\frac{\sum_{i=1}^{n} x_{i}^{2}-\frac{\left(\sum_{i=1}^{n} x_{i}\right)^{2}}{n}}{n-1} \\
\operatorname{Var}_{2}(\hat{x})=\frac{1}{4}\left[\frac{\operatorname{Var}(\hat{b})}{\hat{c}^{2}}+\frac{\hat{b}^{2} \cdot \operatorname{Var}(\hat{c})}{\hat{c}^{4}}\right] \\
\operatorname{Var}_{3}(\hat{x})=\frac{1}{4} \operatorname{Var}\left[\frac{\hat{b}}{\hat{c}}\right]=\frac{1}{4}\left[\left(\frac{\mu_{\hat{b}}}{\mu_{\hat{c}}^{2}}\right)^{2}\left(\frac{\operatorname{Var}[\hat{b}]}{\mu_{\hat{b}}^{2}}+\frac{\operatorname{Var}[\hat{c}]}{\mu_{\hat{c}}^{2}}-\frac{2 \operatorname{Cov}[\hat{b}, \hat{c}]}{\mu_{\hat{b}} \mu_{\hat{c}}}\right)\right] .
\end{gathered}
$$

Para cada situação do conjunto de 5000 simulações, foi obtida a média do ponto crítico e das três variâncias. Do mesmo modo, procedeu-se para o cálculo do coeficiente de assimetria e curtose. Para os coeficientes de assimetria e curtose, calculam-se $\hat{\gamma}_{1}, \hat{\gamma}_{2}$, cujas respectivas variâncias são constantes em todos os casos, visto que todos os vetores têm 5000 observações, assim:

$$
\operatorname{Var}\left(\hat{\gamma}_{1}\right)=0,0012 \quad e \quad \operatorname{Var}\left(\hat{\gamma}_{2}\right)=0,0048
$$

para o cálculo da estatística " $t$ " de Student. Conhecidas as estimativas das variâncias, determinaram-se os intervalos de confiança para $\hat{x}=\frac{\hat{b}}{-2 \hat{c}}$, estimador do ponto crítico, a um coeficiente de confiança de $95 \%$, estimando-se os seus extremos, da seguinte forma:

$$
\overline{\hat{x}} \pm t \sqrt{\operatorname{Var}_{1}(\hat{x})}, \quad \overline{\hat{x}} \pm t \sqrt{\operatorname{Var}_{2}(\hat{x})} \text { e } \overline{\hat{x}} \pm t \sqrt{\operatorname{Var}_{3}(\hat{x})}
$$

em que $\mathrm{t}=2$. Procedeu-se também à construção dos histogramas de freqüência para verificar graficamente se a distribuição dos pontos críticos aproxima-se de uma distribuição normal.

\section{RESULTADOS E DISCUSSÃO}

Com a finalidade de verificar a aplicação das três expressões para o cálculo da variância do ponto crítico e de se ter uma idéia se os valores simulados do ponto crítico seguem uma distribuição normal, são apresentados a seguir os resultados oriundos de várias simulações realizadas. Os resultados obtidos para as estatísticas médias dos coeficientes de regressão $\hat{b}$ e $\hat{c}$, bem como suas respectivas variâncias em função das diversas variân- cias teóricas $\left(\sigma^{2}\right)$ adotadas, estão apresentadas na Tabela 1. Pode-se verificar que os valores médios dos coeficientes de regressão $\overline{\hat{b}}$ e $\overline{\hat{C}}$ estão próximos aos valores teóricos reais considerados. Os valores de $\hat{b}$ variam de 90,1741 a 90,2330 e os valores de $\hat{c}$ variam de $-98,9050$ a 99,1258 . Notou-se uma tendência de que com o aumento da variância teórica, esses valores tiveram um certo aumento. Já para os valores das variâncias de $\hat{b}$ e $\hat{c}$, à medida que a variância teórica $\left(\sigma^{2}\right)$ aumentou, seus valores também aumentaram; nota-se que as variâncias $\operatorname{Var}(\hat{b})$ estão próximas e com tendência na variância teórica $\left(\sigma^{2}\right)$; o mesmo ocorrendo para $\operatorname{Var}(\hat{c})$, numa proporção próxima de 24,45 vezes, o que já era esperado. Os valores das estimativas $\overline{\hat{b}}$ e $\overline{\hat{c}}$, assim como $\operatorname{Var}(\hat{b})$ e $\operatorname{Var}(\hat{c})$, aumentaram à medida que as variâncias teóricas consideradas aumentaram.

Na Tabela 2 apresentam-se os valores médios do ponto crítico e as variâncias obtidas pelos 3 métodos considerados. Pelos resultados, verifica-se que houve um aumento nos valores do ponto crítico à medida que a variância teórica aumentou. Os valores médios do ponto crítico variaram de 0,4560 na variância 0,1 até 0,5586 na maior variância considerada de 50. Observando-se os valores obtidos para as três variâncias, verificou-se que a variância $\operatorname{Var}_{3}(\hat{x})$ apresentou valores menores do que os calculados por $\operatorname{Var}_{1}(\hat{x})$ ou $\operatorname{Var}_{2}(\hat{x})$. Podese inferir por esse que a expressão $\operatorname{Var}_{3}(\hat{x})$, sugerida por Mood, Graybill e Boes (1974), deve ser preferida em vez das outras duas.

Os valores médios obtidos para os coeficientes de assimetria e curtose e respectivos testes " $\mathrm{t}$ " estão apresentados na Tabela 3. Verifica-se que, com o aumento da variância $\left(\sigma^{2}\right)$, houve um aumento do coeficiente de assimetria e de curtose, e os valores de $\hat{\gamma}_{1}$ e $\hat{\gamma}_{2}$ foram todos positivos e aumentaram com o incremento das variâncias teóricas consideradas, evidenciando uma assimetria positiva para todas as variâncias e uma tendência leptocúrtica. 
TABELA 1 - Valores médios dos coeficientes de $\hat{b}$ e $\hat{c}$ e respectivas variâncias em função das diferentes variâncias reais utilizadas.

\begin{tabular}{ccccc}
\hline$\sigma^{2}$ & $\overline{\hat{b}}$ & $\overline{\hat{c}}$ & $\operatorname{Var}(\hat{b})$ & $\operatorname{Var}(\hat{c})$ \\
\hline 0,1 & 90,1741 & $-98,9050$ & 0,1008 & 2,4370 \\
0,5 & 90,1775 & $-98,9178$ & 0,5040 & 12,1851 \\
1 & 90,1800 & $-98,9274$ & 1,0080 & 24,3701 \\
5 & 90,1908 & $-98,9678$ & 5,0402 & 121,8507 \\
10 & 90,1989 & $-98,9981$ & 10,0804 & 243,7013 \\
15 & 90,2051 & $-99,0213$ & 15,1206 & 365,5520 \\
20 & 90,2103 & $-99,0409$ & 20,1608 & 487,4026 \\
\hline 0 & 90,2330 & $-99,1258$ & 50,4020 & 1218,5000 \\
\hline
\end{tabular}

TABELA 2 - Valores médios do ponto crítico $(\overline{\hat{x}})$ e variâncias do ponto crítico calculados pelos métodos: usual $\operatorname{Var}_{1}(\hat{x})$, fórmula deduzida por D’Aulísio (1976) $\operatorname{Var}_{2}(\hat{x})$, expressão dada por Mood et al. (1974) $\operatorname{Var}_{3}(\hat{x})$.

\begin{tabular}{ccccc}
\hline & Ponto crítico & \multicolumn{3}{c}{ Variâncias } \\
\cline { 3 - 4 }$\sigma^{2}$ & $\overline{\hat{x}}$ & $\operatorname{Var}_{1}(\hat{x})$ & $\operatorname{Var}_{2}(\hat{x})$ & $\operatorname{Var}_{3}(\hat{x})$ \\
\hline 0,1 & 0,4560 & 0,000054 & 0,000054 & 0,000001 \\
0,5 & 0,4564 & 0,000274 & 0,000271 & 0,000009 \\
1 & 0,4569 & 0,000555 & 0,000543 & 0,000019 \\
5 & 0,4616 & 0,0030 & 0,0027 & 0,000097 \\
10 & 0,4680 & 0,0069 & 0,0054 & 0,000195 \\
15 & 0,4751 & 0,0123 & 0,0081 & 0,000293 \\
20 & 0,4835 & 0,0211 & 0,0108 & 0,000390 \\
50 & 0,5586 & 2,7808 & 0,0270 & 0,000977
\end{tabular}


Distribuição de freqüência dos valores do ponto crítico

Pode-se observar na Figura 1 que a distribuição de freqüência dos valores do ponto crítico não estão muito distantes dos de uma curva normal. Esse foi o caso em que o coeficiente de curtose se aproximou do de uma normal, não diferindo de 3 ou de zero, quando se subtrai essa constante. Na Figura 2 e de acordo com a Tabela 3, pode-se observar uma leve tendência de assimetria à direita, caracterizando uma distribuição leptocúrtica. Na Figura 3 observa-se uma forte tendência de assimetria à direita e ao formato leptocúrtico (curtose maior que 3), que já havia sido detectada anteriormente nas análises realizadas na Tabela 3.

\section{Intervalos de confiança para o ponto crítico}

Analisando os intervalos de confiança para o ponto crítico calculados usando-se as três diferentes variâncias (Tabela 4), nota-se que esses intervalos são bastante satisfatórios, principalmente quando se utilizou a variância $\operatorname{Var}_{3}(\hat{x})$. Como já era esperado, todos os intervalos de confiança tornaram-se menos precisos com o aumento das variâncias teóricas. Desse modo, os intervalos de confiança menos satisfatórios foram aqueles obtidos com $\operatorname{Var}_{1}(\hat{x})$ e os intervalos mais satisfatórios, obtidos com $\operatorname{Var}_{3}(\hat{x})$.

TABELA 3 - Valores médios dos coeficientes de assimetria, curtose e respectivas estatísticas do teste " $t$ ", em função das diferentes variâncias.

\begin{tabular}{ccccc}
\hline & Coeficiente de assimetria & Coeficiente de curtose & \multicolumn{2}{c}{ Valores do teste “ $t$ ”' } \\
\cline { 4 - 5 }$\sigma^{2}$ & $\hat{\gamma}_{1}$ & $\hat{\gamma}_{2}$ & $t_{\hat{\gamma}_{1}}$ & $t_{\hat{\gamma}_{2}}$ \\
\hline 0,1 & 0,0962 & 0,1311 & $2,7775^{(* *)}$ & $1,8928^{(\mathbb{N S})}$ \\
0,5 & 0,2197 & 0,2281 & $6,3447^{(* *)}$ & $3,2947^{(* *)}$ \\
1 & 0,3157 & 0,3478 & $9,1163^{(* *)}$ & $5,0232^{(* *)}$ \\
5 & 0,7906 & 1,5481 & $22,8301^{(* *)}$ & $22,3559^{(* *)}$ \\
10 & 1,3329 & 4,3136 & $38,4902^{(* *)}$ & $62,2927^{(* *)}$ \\
15 & 2,1221 & 11,2430 & $61,2783^{(* *)}$ & $162,3599^{(* *)}$ \\
20 & 3,8834 & 37,6100 & $112,1372^{(* *)}$ & $543,1243^{(* *)}$ \\
50 & 12,4604 & 1277,7000 & $359,8073^{(* *)}$ & $18451,0000^{(* *)}$ \\
\hline
\end{tabular}

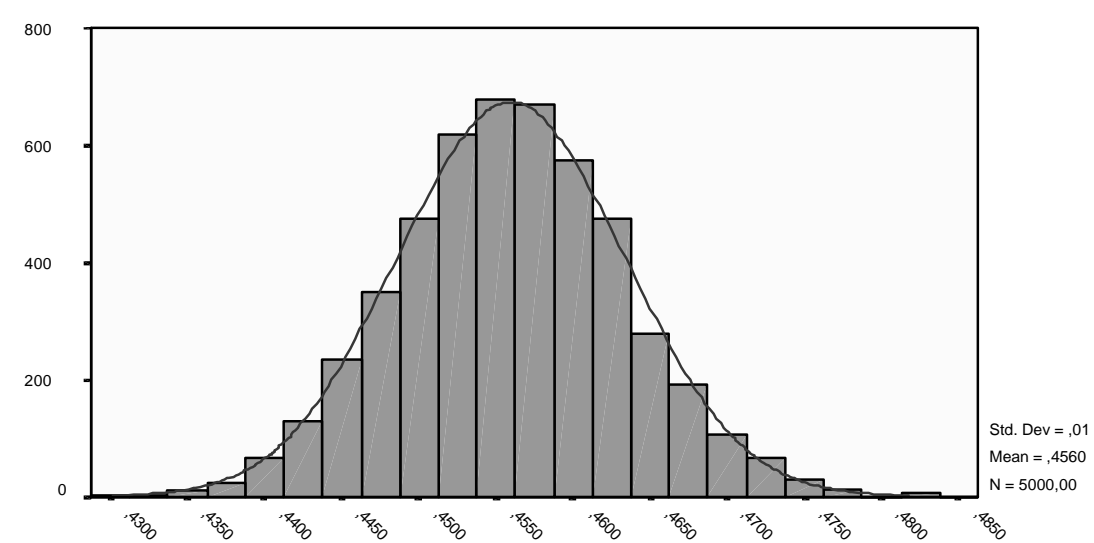

FIGURA 1 - Representação gráfica da distribuição de frequiência dos valores simulados do ponto crítico, para a variância $\sigma^{2}=0,1$ e valor médio de $\overline{\hat{x}}=0,4560$. 


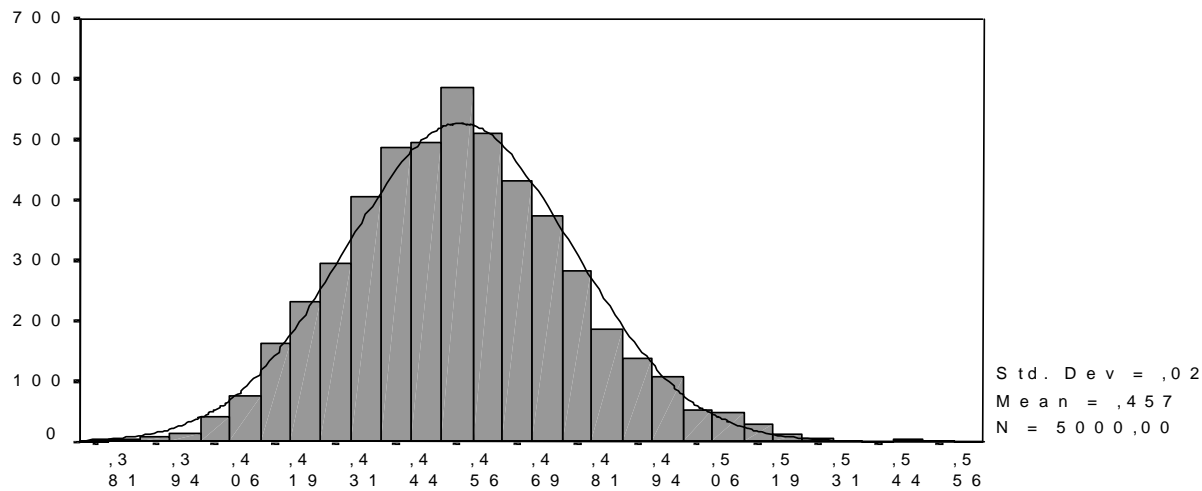

FIGURA 2 - Representação gráfica da distribuição de frequiência dos valores simulados do ponto crítico, para a variância $\sigma^{2}=1,0$ e valor médio de $\overline{\hat{x}}=0,4569$.

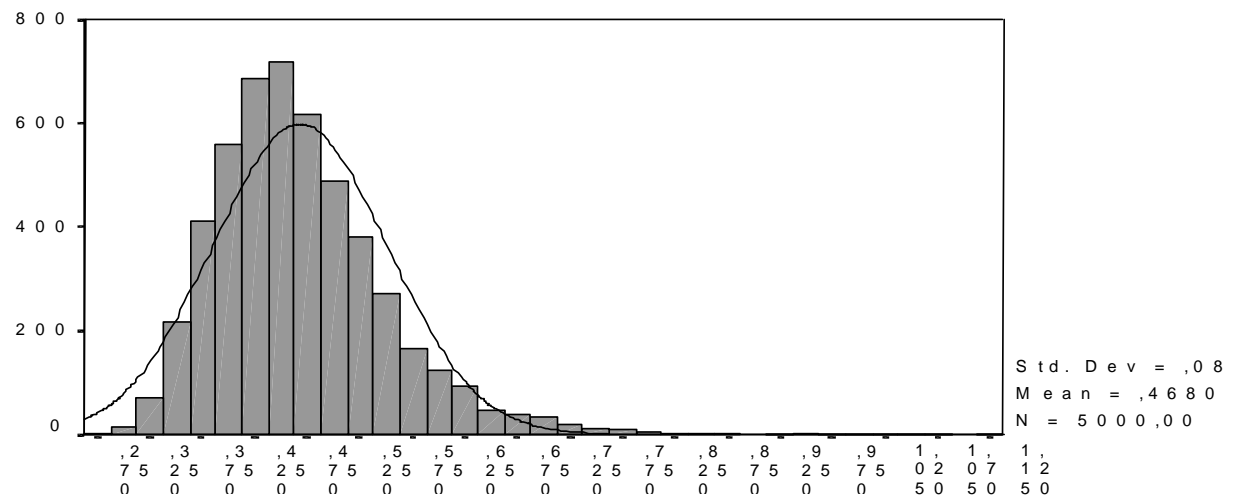

FIGURA 3 - Representação gráfica da distribuição de frequiência dos valores simulados do ponto crítico, para a variância $\sigma^{2}=10,0$ e valor médio de $\overline{\hat{x}}=0,4680$.

TABELA 4 - Valores médios do ponto crítico e intervalos de confiança para o ponto crítico considerando as três variâncias, em função das variâncias teóricas.

\begin{tabular}{ccccc}
\hline Variância teórica & Ponto crítico médio & \multicolumn{3}{c}{ Variâncias } \\
\cline { 3 - 5 }$\left(\sigma^{2}\right)$ & $(\overline{\hat{x}})$ & $\operatorname{Var}_{1}(\hat{x})$ & $\operatorname{Var}_{2}(\hat{x})$ & $\operatorname{Var}_{3}(\hat{x})$ \\
\hline 0,1 & 0,4560 & {$[0,4412 ; 0,4707]$} & {$[0,4412 ; 0,4707]$} & {$[0,4532 ; 0,4588]$} \\
0,5 & 0,4564 & {$[0,4232 ; 0,4896]$} & {$[0,4234 ; 0,4894]$} & {$[0,4501 ; 0,4626]$} \\
1 & 0,4569 & {$[0,4098 ; 0,5041]$} & {$[0,4103 ; 0,5035]$} & {$[0,4481 ; 0,4658]$} \\
5 & 0,4616 & {$[0,3514 ; 0,5718]$} & {$[0,3574 ; 0,5657]$} & {$[0,4418 ; 0,4813]$} \\
10 & 0,4680 & {$[0,3016 ; 0,6343]$} & {$[0,3208 ; 0,6152]$} & {$[0,4400 ; 0,4959]$} \\
15 & 0,4751 & {$[0,2535 ; 0,6968]$} & {$[0,2949,0,6554]$} & {$[0,4409 ; 0,5094]$} \\
20 & 0,4835 & {$[0,1929 ; 0,7742]$} & {$[0,2755 ; 0,6916]$} & {$[0,4440 ; 0,5231]$} \\
50 & 0,5586 & {$[-2,7765 ; 3,8937]$} & {$[0,2301 ; 0,8871]$} & {$[0,49610,6211]$} \\
\hline
\end{tabular}

Ciênc. agrotec., Lavras, v. 28, n. 2, p. 389-396, mar./abr., 2004 


\section{CONCLUSÕES}

As variâncias dos coeficientes de regressão aumentaram proporcionalmente com o aumento das variâncias teóricas $\left(\sigma^{2}\right)$ consideradas, caracterizando uma simulação de boa qualidade. A variância do ponto crítico calculada usando-se a expressão de Mood et al. (1974) apresenta-se como a mais satisfatória, pois leva em consideração a covariância entre os coeficientes de regressão $b$ e $c$. A variância $\sigma^{2}$ afeta a estimativa do ponto crítico, maiores variâncias estão relacionados com maiores valores médios. A distribuição de freqüência do ponto crítico não segue ao de uma distribuição normal. $\mathrm{O}$ ponto crítico apresenta uma distribuição de freqüência com assimetria positiva e formato do tipo leptocúrtico. Os intervalos de confiança para o ponto crítico tornam-se menos precisos com o aumento das variâncias teóricas $\left(\sigma^{2}\right)$ em todos os três casos analisados. Os intervalos de confiança com menor amplitude foram os obtidos utilizando-se a variância calculada por $\operatorname{Var}_{3}(\hat{x})$.

\section{REFERÊNCIAS BIBLIOGRÁFICAS}

D’AULISIO, M. B. G. d'. A variância dos pontos de máximo ou de mínimo de equações de regressão de segundo grau. 1976. 61 f. Dissertação (Mestrado em Estatística e Experimentação Agronômica) - Escola Superior de Agricultura de "Luiz de Queiroz", Piracicaba, 1976.
FINNEY, D. J. Statistical method in biological assay. London: Charles Griffin, 1964. 668 p.

FISHER, R. A. The moments of the distribution for normal samples of measures of departure from normality. Journal of the Royal Statistical Society, A, Londres, v. 130, p. 17-28, 1930.

FREITAS, A. R. A distribuição do ponto de máximo ou de mínimo de uma função usada em experimentos de adubação. 1978. 81 f. Dissertação (Mestrado em Estatística e Experimentação Agronômica) - Escola Superior de Agricultura de "Luiz de Queiroz", Piracicaba, 1978.

KAPLAN, W. Cálculo avançado. São Paulo: Edgard Blücher, 1972. v. 2, 750 p.

KENDALL, M. G.; STUART, A. The advanced theory of statistics. London: Charles Griffin, 1977. v. 1.

MOOD, A. M.; GRAYBILL, F. A.; BOES, D. C. Introduction to the theory of statistics. 3. ed. Tokio: McGraw-Hill, 1974. 564 p.

NETER, J.; WASSERMAN, W.; KUTNER, M. H. Applied linear statistical models: regression, analysis of variance, and experimental designs. 3. ed. Homewood: Richard D. Irwin, 1990. 1181 p.

SPIEGEL, M. R. Estatística. 3. ed. São Paulo: Makron Books, 1993. 643 p. 